\title{
Präexpositionsprophylaxe
}

\section{HIV-Therapie auch für Nichtinfizierte}

Rechtzeitig verabreicht lässt sich mit modernen Arzneien postexpositionell eine HIV-Infektion verhindern, in bestimmten Fällen auch präexpositionell.

Die neuen Möglichkeiten der medikamentösen Intervention gegen den AIDSErreger HIV haben auch Einfluss auf die postexpositionelle Prophylaxe (PEP) und eröffnen sogar die Option, bereits präexpositionell vorzugehen.

\section{PEP bei Kontakt mit Risikomaterial} Die aktuelle, bis 2017 gültige deutsch-österreichische Leitlinie zur postexpositionellen Prophylaxe der HIV-Infektion empfiehlt eine medikamentöse Prophylaxe, wenn es Kontakte mit erhöhtem Infektionsrisiko gegeben hat.Als solche gelten die perkutane Stichverletzung mit einer Injektionsnadel oder einer anderen Hohlraumnadel sowie eine Schnittverletzung unter Beteiligung von Körperflüssigkeiten mit potenziell hoher HIV-Konzentration, wie es in der Leitlinie heißt.

Die PEP könne angeboten werden, wenn ein Schleimhautkontakt oder Kontakt mit nicht-intakter Haut - z. B bei einem Hautekzem oder einer frischen Wunde -, mit Flüssigkeiten von hoher Viruskonzentration oder bei sichtbaren Verletzungen, etwa mit einer blutig-tingierten chirurgischen Nadel, vorliegt. Bei perkutanem Kontakt mit anderen Körperflüssigkeiten als Blut (etwa Urin oder Emtricitabin plus Tenofovir genannt. für eine PEP wird allerdings abgeraten.
Speichel), bei Kontakt von intakter Haut mit Blut (auch bei hoher Viruskonzentration) sowie bei Haut- oder Schleimhautkontakt mit Körperflüssigkeiten wie Urin und Speichel ist eine PEP nicht in $>$ diziert.

Eine PEP sollte so früh wie möglich begonnen werden, am besten innerhalb von zwei Stunden oder spätestens nach 24 Stunden, und über 28 - 30 Tage vorgenommen werden. Als Beispiel für eine medikamentöse Prophylaxe wird in der Leitlinie die zweimal täglich einzunehmende Kombination des Integrasehemmers Raltegravir mit der Fixkombination

Primäre Alternative zu Raltegravir sei der Proteasehemmer Lopinavir, zur Fixkombination ein Präparat mit Zidovudin plus Epivir. Von Abacavir und Nevirapin

\section{PrEP bei HIV-Negativen mit hohem Infektionsrisiko}

Spätestens seit den entsprechenden WHOEmpfehlungen im Herbst 2015 ist auch die präexpositionelle HIV-Prophylaxe (PrEP) zum Thema geworden. Der vorläufigen Leitlinie zufolge wird eine tägliche orale Prophylaxe als Teil einer Kombinationstherapie HIV-negativen Personen mit sehr

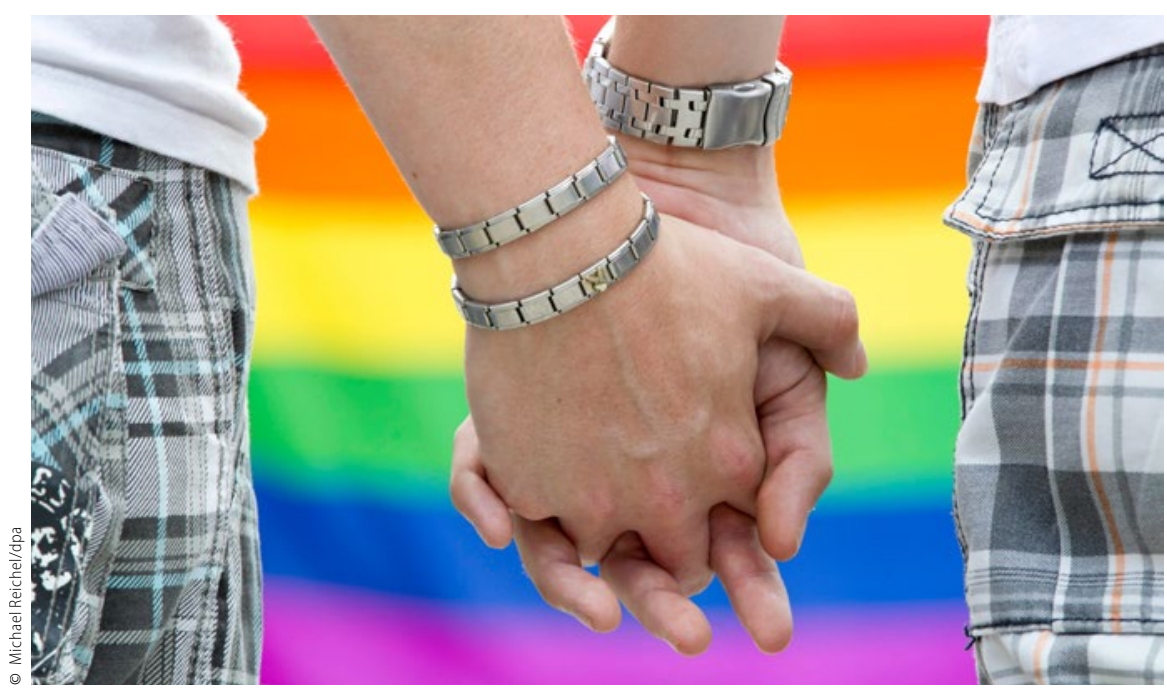

Männer, die Sex mit Männern haben, gehören aufgrund ihres hohen Infektionsrisikos zur Zielgruppe für eine präexpositionelle HIV-Prophylaxe. hohem Infektionsrisiko generell empfohlen, ohne sich auf eine bestimmte Bevölkerungsgruppe, etwa Männer, die Sex mit Männern haben, zu beschränken.

\section{Infektionsrisiko um 92\% reduziert}

Die Empfehlung stützt sich auf Studienergebnisse, denen zufolge die antiretrovirale Behandlung mit Tenofovir zur PrEP wirksam vor einer HIV-Infektion schützt. Die Wahrscheinlichkeit, dass sich Menschen mit einem erhöhten Infektionsrisiko anstecken, wird bei regelmäßiger Medikamenteneinnahme um $92 \%$ reduziert. Studien bestätigten, dass durch eine solche Prophylaxe der Gebrauch von Kondomen nicht geringer wird. Zu betonen ist, dass die HIVPrEP nur als zusätzliche Option zu anderen Maßnahmen, sich vor einer Infektion zu schützen - also safer sex -, betrachtet wird.

In Deutschland ist - anders als etwa in den USA und Frankreich - Tenofovir derzeit nicht zur HIV-PrEP zugelassen, sondern nur zur Therapie bei HIV-Infizierten. Wo es zur PrEP zugelassen ist, ist die Substanz Teil eines Kombinationspräparats mit Emtricitabin.

Die europäische Zulassung der Fixkombination Tenofovirdisoproxil plus Emtricitabin zur PrEP wird derzeit von der European Medicines Agency (EMA) geprüft, und zwar auf der Grundlage der Ergebnisse der beiden Studien „Pre-Exposure Prophylaxis Initiative" (iPrEX) und „Partners PrEP“.

(Peter Leiner)

Leitlinie "HIV-Infektion, postexpositionelle Prophylaxe", AWMF-Nr:: $055-004$

\section{INFOS IM INTERNET}

Alle Folgen der Sommer-Akademie finden Sie im Web unter www.aerztezeitung.de

Auf www.daignet.de präsentiert sich die Deutsche AIDS-Gesellschaft; sie setzt sich für die nichtkommerzielle und universitätsnahe Forschung zur Optimierung der HIV-Therapie ein.

Die Deutsche Arbeitsgemeinschaft niedergelassener Ärzte in der Versorgung HIV-Infizierter bietet auf www.dagnae.de Infos etwa zu wichtigen Leitlinien und Qualitätsmanagement.

Die Europäische AIDS-Gesellschaft EACS bietet unter www.eacsociety.org Zugriff auf Leitlinien und Kongressmaterial. 